\title{
Comparative Study between Intracavernosal Injection of Botulinum Toxin type A [50 and 100 unit], Efficacy and Durability in the Treatment of Vascular Erectile Dysfunction
}

\author{
A.A.Taleb ${ }^{1}$, M.El-Tabey ${ }^{1}$ W.A.El-Shaer ${ }^{1}$, H.M.Ghanem ${ }^{2}$ and T.A.wahab ${ }^{1}$ \\ ${ }^{1}$ Urology Dept, Faculty of Medicine, Benha Univ, Benha, Egypt \\ ${ }^{2}$ Andrology Dept, Faculty of Medicine, Cairo Univ, Egypt \\ E-Mail: Tamerdiab100@gmail.com
}

\begin{abstract}
Evidence has been arising suggesting that Botulinum toxin type A [BTX-A] injections can relax smooth muscles fibers in the treatment of obesity and Detrusor muscle over-activity, similar effect on cavernosal smooth muscles would help in the treatment of erectile dysfunction [ED] resistant to oral and intracavernous injection [ICI] therapy, thus it could be an alternative option for failed medical and other injectable therapy.. to compare safety, efficacy and durability of different doses of BTX-A [50 and 100 Unit] in the treatment of Vasculogenic Erectile Dysfunction after failure of other ICI therapy.. Forty-five patients with Vasculogenic erectile dysfunction who are failed treatment with non-surgical options were included at this study after elimination of all patients who are not matching eligible criteria or not complete the follow up program. The patients were randomized into 3equal groups, each group include 15 patients. Group [I] injected with 100U BTX-A, group [II] injected with 50U BTX-A and Control group injected with normal saline. All groups underwent follow up at 2 weeks, 3 months and 6 months intervals after injection, for assessment of all subjective data as, IIEF-5 Questionnaire [SHIM score], Erection hardness score [EHS], Sexual encounter profile [SEP 2 and 3], Global assessment score[ GAS 2 and 3]. Also assessment of objective data by penile Doppler details.. Although 25 patients [55.6\%] respond to BTX-A injection subjectively and objectively \{13 patients [28.9\%] in group [I] and 12 patients [26.7\%] in group [II]\}, but this impressive effect was not durable especially in group [II] as 9 patients [20\%] show lost effect through complete follow up till 6 month in comparison to 5 patients [11.1\%] in group [I] show same lost effect. Besides that, 5 patients [11.1\%] not responding to this treatment option \{two patients [4.4\%] in group [I] and 3 patients [6.7\%] in group [II], also patients in Control group show non-significant subjective improvement. BTX-A could be a potential but not durable therapy for erectile dysfunction, as BTX-A [50 and 100 Unite] show almost same efficacy but, with complete follow up through 6 months, BTX-A 100U is more durable than $50 \mathrm{U}$.
\end{abstract}

Keywords: Intracavernosal injection, Botulinum Toxin type A [BTX-A], Vascular Erectile dysfunction, Efficacy and Durability.

\section{Introduction}

\subsection{Erectile dysfunction}

Erectile dysfunction [ED] is widely accepted now as a persistent inability to achieve or maintain a penile erection sufficient to permit satisfactory sexual performance, reflecting a dramatic effect on quality of life for the man and his partner [1-7].

ED commonly occurs, and it's incidence increases with age, its prevalence is anticipated to increase from 152 million in 1995 to 322 million by 2025 [8-9].

ED is classified as psychogenic, organic, or mixed psychogenic and organic, $80 \%$ of cases are now considered to be organic in origin, but the final common pathway in the majority of subjects with organic ED is endothelial dysfunction[vasculogenic ED] [10-11].

Subjective and objective assessment should included at initial evaluation, medical, sexual, and psychosocial histories, as well as laboratory assays and complete Doppler study to identify possible comorbidities for a wellorganized management [12-14].
Fifty years ago, psychotherapy was the mainstay treatment and undoubtedly was limited in its success. During the 1970s, penile prostheses combined with psychotherapy remained popular but relatively inaccessible. In the 1980s, intracavernosal injection [ICI] emerged, followed by intra-urethral therapy in the mid-1990s. The true revolution in the nonsurgical management of ED was with the introduction of oral phosphodiesterase type 5 inhibitors [PDE5Is] in the late 1990s and subsequently. PDE5Is rapidly became the patient-friendly method of ED treatment and are currently considered as first-line monotherapy 15].

ICI has been used successfully since 1982 for patients with erectile dysfunction. Despite advances in oral and intraurethral treatments, ICI remains a very effective second line therapy for many patients [16-18].

Recently, the possibility of using stem cells or gene therapy has shown some early promise but disappointing clinical outcomes [19].

\subsection{Botulinum toxin}


One of the most potent toxins known to humans produced by Clostridium botulinum, resulting in generalized paralysis including respiratory arrest and death, is Botulinum neurotoxin [BoNT] [20-21].

Although seven biochemical and serologic forms of BoNT [A, B, C1, D, E, F, and G] are known, only BoNT-A, BoNT-B, and BoNT-E can cause botulism in humans, whereas the remaining BoNT forms can cause disease only in animals. BoNT-A is the most commonly used form in medicine [22-23].

One of the newest putative therapeutic opportunities to be explored is the intracavernosal delivery of botulinum toxin A for ED. Most clinicians and the general public are familiar with its utility in dermatology suites and plastic surgery facial clinics for cosmetic indications. Intra detrusor delivery of Botox has been approved for patients with neurogenic and idiopathic detrusor overactivity. The potential for its use in facilitating long-acting cavernous smooth muscle relaxation through alteration of the balance within the penis between the permanent sympathetic contractile tone applied to cavernosal smooth muscle fibers responsible for flaccidity and the relaxed state of the cavernous smooth muscles induced by the activation of the parasympathetic nitric oxide [NO]-cyclic guanosine phosphate pathway in erection is a potential game changer [24].

Botulinum toxin A can inhibit norepinephrine, but not NO, release Thus, Botulinum toxin A could be responsible for a persisting modulatory effect on the erectile tissue facilitating an erectile response to sexual stimulation [19]

Currently, few real data on this pathway or treatment approach exist apart from a small basic animal study by De-Young et al25, and a pilot study from Egypt by Ghanem et al [26].

\section{Patients and Methods}

This was a prospective randomized doubleblind placebo controlled study conducted in the outpatient setting. Ethical approval was obtained from the Institutional Ethics Committee before beginning.

\subsection{Eligibility}

Forty five patients with ranged age between 40 to 70 years old proved to be Vasculogenic ED by Penile Duplex, unable to develop erections sufficient for intercourse with "No" response on Sexual Encounter Profile questions [SEP $2 \&$ 3], failing to respond to first and second line treatments for ED, and surgery is the only remaining treatment option for them were eligible for inclusion in our study Contrarily, patients with significant cardiovascular disease interfering with sexual activity, any history of an unstable psychiatric conditions and presence of penile anatomical abnormalities that would significantly impair erectile function were excluded from this study

\subsection{Baseline evaluation}

All patients were assessed basally by full sexual history with scoring evaluation contained through, Sexual Health Inventory for Men score [SHIM], Erection Hardness Score [EHS], Sexual Encounter Profile [SEPQuestions 2 and 3] and Global Assessment Score [GAS]., then local penile examination to exclude any anatomical abnormalities followed by initial penile Doppler using a trimix solution [PGE1 $10 \mathrm{ug}+$ Phentolamine 1 $\mathrm{mg}+$ Papaverine $30 \mathrm{mg}$ ], finally all data were recorded to be followed up.

\subsection{Randomization and allocation}

Those patients equally randomized into 3 groups I, II. Injected by 100 and 50Unit BTXA, and Control group injected with normal saline.

\subsection{Procedure}

In a recumbent position at controlled airconditioned environment $\left[24{ }^{\circ} \mathrm{C}\right]$, and promptly after the patient stripp of his clothes to minimize thermal effect, a fully stretched flaccid penile length which considered equivalent to erected penile length as described by Wessels et al27 was measured by same doctor using a tape from pubo-penile junction to distal glans, also a rigid ruler was applied to push suprapubic fat to confirm measurement and decrease inaccurate measures in obese patients, beside that, a midshaft penile girth was obtained using a rubber tape and all measures were approximated to the nearest $0.5 \mathrm{~cm}$.

A rubber band was tied at the root of penis, skin was sterilized by alcohol $70 \%$ spray followed by application of povidone-iodine using a sterile gauze.

Botox [Onabotulinum toxin A; Allergan, Irvine, Ireland] diluted in isotonic saline injected through an insulin syringe intracavernosally $2 \mathrm{~cm}$ proximal to coronal margin and $2 \mathrm{~cm}$ distal to pubo-penile junction at right and left sides respectively followed by a fine massage at four sites of injection for 5 minutes and the band removed after 20 minutes.

\subsection{Follow up}

All patients in each group were evaluated at the following interval, 2 weeks, 3 months and 6 months after Botox injection subjectively and 
objectively as the same method described in basal assessment.

\section{Results}

Table (1) Three Groups Through Timeframe Evaluation

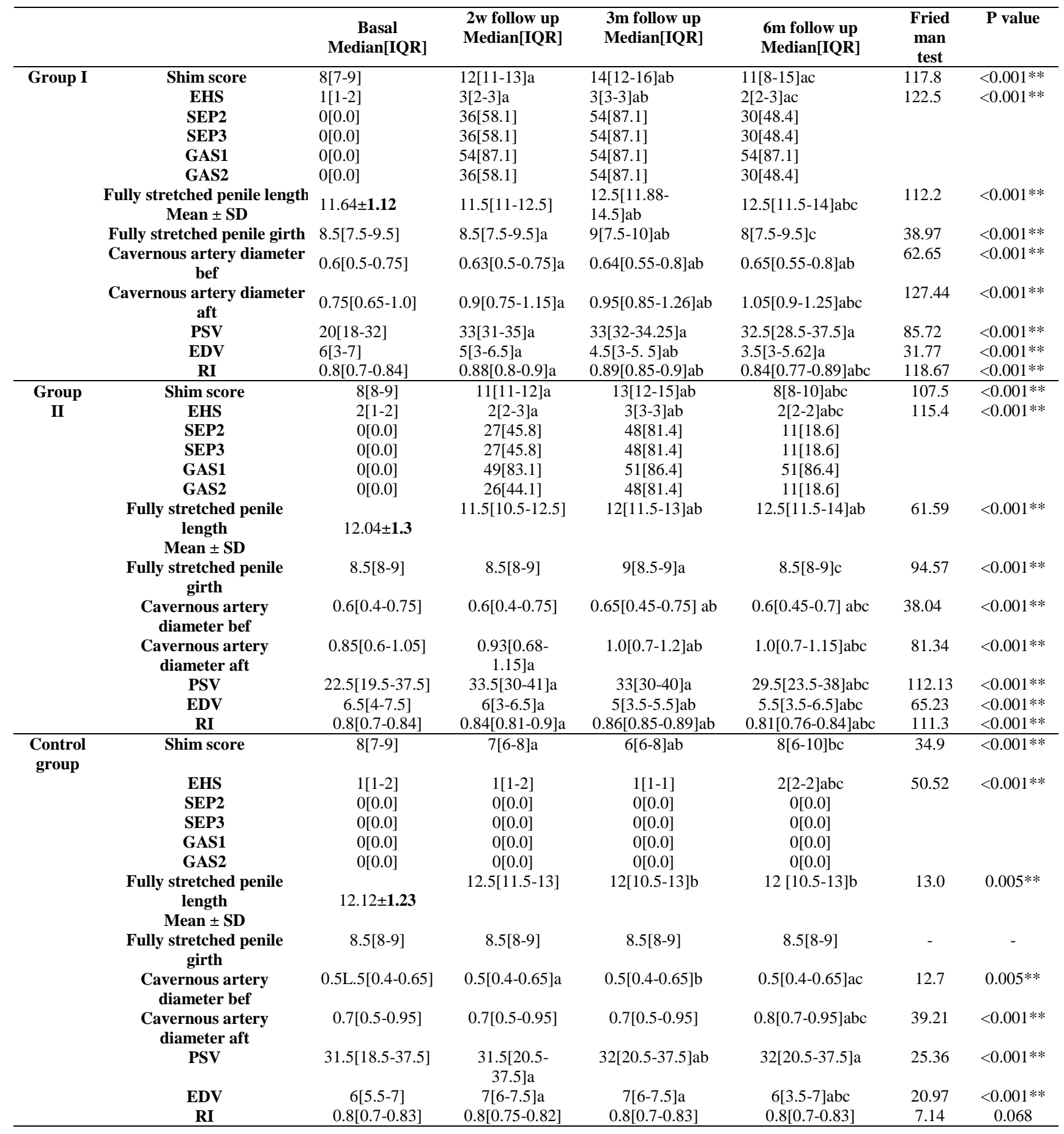

Although 25 patients [55.6\%] respond to BTX-A injection subjectively and objectively $\{13$ patients [28.9\%] in group [I] and 12 patients [26.7\%] in group [II]\}, but this impressive effect was not durable especially in group [II] as 9 patients [20\%] show lost effect through complete follow up till 6 month in comparison to 5 patients [11.1\%] in group [I] show same lost effect. Besides that, 5 patients [11.1\%] not responding to this treatment option \{two patients [4.4\%] in group [I] and 3 patients [6.7\%] in group $[\mathrm{II}]\}$, also patients in Control group show non-significant subjective improvement. 


\section{Discussion}

As ED has a strong negative impact on quality of life and human psychological comfort $[\mathbf{2 8 , 2 9}]$ and it is believed that the disease is always hidden with insufficient treatment so, it was necessary finding a new trends in treatment [30].

Where cavernosal smooth muscles relaxation is the corner stone in erection physiology which is mediated by nitric oxide production resulting from stimulation of parasympathetic cholinergic and nonadrenergic non-cholinergic neurons.31so, sinusoidal smooth muscle relaxation effect of BoNT-A produce rational concept about its role in treatment.

Our belief in this treatment pathway was settled down after previous experiences of a small basic animal study by De Young et al[25] on old rats receiving Botox $10 \mathrm{U}$ compared with controls and small human pilot study by Ghanem et al [26] which 12 patients received a single intracavernous injection of Botox $50 \mathrm{U}$ with increased arterial flow and improved SHIM score after 2 weeks.

Our results confirm the results of Ghanem et al [26] in safety concern of Botox and its effective role in treatment of patients with refractory $\mathrm{ED}$, beside that with application comparison between 50 and $100 \mathrm{U}$ of BoNTA, our results show same efficacy of $100 \mathrm{U}$ than $50 \mathrm{U}$ at initial follow up till 3 months but, with complete follow up till 6 months, BoNTA $100 \mathrm{U}$ show more durable action than $50 \mathrm{U}$ with same safety.

\section{Conclusion}

BTX-A could be a potential but not durable therapy for erectile dysfunction, as BTX-A [50 and 100 Unite] show almost same efficacy but, with complete follow up through 6 months, BTX-A $100 \mathrm{U}$ is more durable than $50 \mathrm{U}$.

\section{References}

[1] R.Lewis, D.Hatzichristou, E. Laumann, J.McKinlay, Epidemiology and natural history of erectile dysfunction: risk factors including iatrogenic and aging. In: Jardin A, Wagner G, Khoury S, Giuliano F, Padma-Nathan H, Rosen R, editors. Erectile dysfunction. Plymouth, UK: Health Publications, Vol.2000, PP.19-51,2014.

[2] T.D.Strong, M.A.Gebska, H.C.Champion, A.L.Burnett, Stem andendothelial progenitor cells in erection biology. Int $\mathbf{J}$ Impot Res, Vol.20,PP.243-54,2008.

[3] J.Tomlinson, D.Wright, Impact of erectile dysfunction and its subsequent treatment with sildenafil: qualitative study. BMJ,Vol.21,PP.328-1037,2004.

[4] M.S.Litwin, R.J.Nied, N.Dhanani, Healthrelated quality of life in men with erectile dysfunction. J Gen Intern Med,Vol.131,PP.59-66,1998.

[5] J.J.Sgnchez-Cruz, A.Cabrera-Len, A. Martdn-Morales, Male erectile dysfunction and health-related quality of life. Eur Urol,Vol.44,PP.245-253,2003.

[6] E.A.Jannini, N.Sternbach, E. Limoncin, Health-related characteristics and unmet needs of men with erectile dysfunction: a survey in five European countries. J Sex Med,Vol.11,PP.40-50,2014.

[7] P.J.DiMeo, Psychosocial and relationship issues in men with erectile dysfunction. Urol Nurs, Vol.26,pp442-446;453,2006.

[8] J.B.McKinlay, The worldwide prevalence and epidemiology of respective PDEs, could achieve greater enhance-erectile dysfunction. Int J Impot Res,Vol.2,PP.7884,2000 .

[9] H.A.Feldman, I.Goldstein, D.G. Hatzichristou, R.J. Krane, Impotence and its medical and psychosocial correlates: results of the Massachusetts male aging study. J Urol,Vol.151,PP.54-61,1994.

[10] R.Balon, R.T.Segraves, Clinical manual of sexual disorder. In: Riley A, Riley E, editors. Male erectile disorder. Washington, DC: American Psychiatric Publish ing,Vol.33,PP.213-49,2009.

[11] M.E.Sullivan, C.S. Thompson, M.R. Dashwood, Nitric oxide and penile erection: Is erectile dysfunction another manifesta tion of vascular disease? Cardiovasc Res,vol,43, pp,658-665,1999.

[12] R.C.Rosen, J.C.Cappelleri, Smith MD, Lipsky J, Peã BM. Development and evaluation of an abridged, 5-item version of the International Index of Erectile Dysfunction [IIEF-5] as a diagnostic tool for erectile dysfunction. Int $\mathbf{J}$ Impot Res,Vol.11,PP.319-26,1999.

[13] R.Ramanathan, J.Mulhall, S.Rao, R.Leung, J.I.Martinez Salamanca, A.Mandhani, Predictive correlation between the International Index of Erectile Function [IIEF] and Sexual Health Inventory for Men [SHIM]: implications for calculating a derived SHIM for clinical use. J Sex Med,Vol.4,PP.1336-44,2007.

[14] I.Goldstein, J.P.Mulhall, A.G. Bushmakin, J.C.Cappelleri, The erection hardness score and its relationship to successful sexual inter course. J Sex Med,Vol.5,PP.2374-80,2008.

[15]T.Lue, G.Broderick, Evaluation and nonsurgical management of erectile 
dysfunction and premature ejaculation. In: Walsh PC, Retik AB, Vaughan ED, Wein AJ, editors. Campbell's urology. 9th ed. Philadelphia, PA: WB Saunders, Vol.52 PP.750-87,2006.

[16] S.J.Beer, W.A.See, Intracorporeal needle breakage: an unusual complication of papaverine injection therapy for impotence. J Urol,Vol.147[1],PP.148$150,1992$.

[17]F.Iacono, S.Barra, Intracorporeal needle breakage as an unusual complication of intracavernous self-injection. Tech Urol,Vol.4[1] ,PP.54- 55,1998.

[18]R.Shamloul, I.Kamel, A broken intracavernous needle: successful ultrasound-guided removal. J Sex Med,Vol.2[1],PP.147-148,2005.

[19] Francois Giuliano, MD, PhD, and Gerald Brock, Botox for Erectile Dysfunction. J Sex Med. ,Vol.14[2],PP.177-178,2017.

[20] K.R.Aoki,Physiology and pharmacology of therapeutic botuli Num neurotoxins. Curr Probl Dermatol,Vol.30,PP.107-116. ,2002.

[21]E.Chung, Botulinum toxin in urology a review of clinical potential in the treatment of urologic and sexual conditions. Expert Opin Biol Ther,Vol.15,PP.95-102,2015

[22] K.R.Aoki, Pharmacology and immunology of botulinum toxin serotypes. J Neurol,Vol.248[1],pp3-10. 2001.

[23] Y.C.Chuang, C.C.Huang, H.Y.Kang, Novel action of botulinum toxin on the stromal and epithelial components of the prostate gland. J Urol,Vol.175,PP.11581163,2006 .
[24] Francois Giuliano, Gerald Brock, Botox for Erectile Dysfunction. J Sex Med.,Vol.14[2],PP.177-178,2017.

[25]L.De Young, J.Campbell, S.Radomski, Intracavernosal injection of botulinum toxin to improve erectile function in older rats. Paper presented at: 20th World Meeting on Sexual Med icine of the International Society of Sexual Medicine. Beijing,China., Vol.45[2],PP.244252,2016.

[26]H.Ghanem, I. Soliman, M .AbdulHamid, Can intracavernosal botulinum toxin injection salvage vascular erectile dysfunction patients not responding to oral and intracavernous therapy? A pilot study. J Sex Med,Vol.13[1],PP.111-116,2016.

[27] H.Wessells, T.F.Lue, J.W. McAninch, Penile length in the flaccid and erect states; guidelines for penile augmentation. J Urol,Vol.156,PP.995-7,1996.

[28]F.Montorsi, G. Adaikan, E .Becher, Summary of the recommendations on sexual dysfunctions in men. J Sex Med,Vol.7,PP.3572-3588,2010

[29] J.J. Sánchez-Cruz, A.Cabrera-León, A.Martín-Morales, Male erectile dysfunction and health-related quality of life. Eur Urol,Vol.44,PP.245-253,2003.

[30]M.Frost, K.Wraae, C.Gudex, Chronic diseases in elderly men: underreporting and underdiagnosis. Age Ageing,Vol.41,pp177-183,2012.

[31] J.Chen, A.Gefen, A.Greenstein, H.Matzkin, D.Elad, Predicting penile size during erection. Int J Impot Res,Vol.12,PP.314-8,2000. 